\title{
Phosphorus doping and hydrogen passivation of donors and defects in silicon nanowires synthesized by laser ablation
}

\author{
N. Fukata, a),b) J. Chen, and T. Sekiguchi \\ Advanced Electronic Materials Center, National Institute for Materials Science, 1-1 Namiki, \\ Tsukuba 305-0044, Japan \\ S. Matsushita, T. Oshima, N. Uchida, and K. Murakami ${ }^{\text {b) }}$ \\ Institute of Applied Physics, University of Tsukuba, 1-1-1 Tennodai, Tsukuba 305-8573, Japan \\ T. Tsurui and S. Ito \\ Institute for Materials Research, Tohoku University, Sendai 980-8577, Japan
}

(Received 14 January 2007; accepted 9 March 2007; published online 12 April 2007)

\begin{abstract}
Phosphorus (P) doping was performed during the synthesis of silicon nanowires (SiNWs) by laser ablation. At least three types of signals were observed by electron spin resonance (ESR) at $4.2 \mathrm{~K}$. Phosphorus doping into substitutional sites of crystalline Si in SiNWs was demonstrated by the detection of an ESR signal with a $g$ value of 1.998, which corresponds to conduction electrons in crystalline $\mathrm{Si}$, and by an energy-dispersive $\mathrm{x}$-ray spectroscopy spectrum of the $\mathrm{P} K \alpha$ line. The ESR results also revealed the presence of defects. These defects were partially passivated by hydrogen and oxygen atoms. (C) 2007 American Institute of Physics. [DOI: 10.1063/1.2721377]
\end{abstract}

The doping and activation of carriers in silicon nanowires (SiNWs) is a key step in realizing nanoscale silicon devices using SiNWs. The vapor-liquid-solid mechanism has been widely used for growing SiNWs, and carrier doping has been performed during growth. The doping effect has been investigated by electrical transport measurements. ${ }^{1-7}$ To evaluate the electrical properties of $\mathrm{SiNWs}, \mathrm{Si} / \mathrm{SiO}_{2}$ interface defects such as $P_{b}$ centers and other impurities need to be investigated, since compensation by interface defects and contamination by metal catalysts need to be considered. On the other hand, it is possible to distinguish information on boron as a dopant from other types by optical measurements. We recently reported a result for boron (B) doping in SiNWs, based on spectroscopic results, such as the observation of the local vibrational mode of B and the Fano effect due to heavy B doping in SiNWs by Raman scattering measurements. ${ }^{8}$ In the case of phosphorus $(\mathrm{P})$ doping, the mass of $\mathrm{P}$ atoms and that of Si atoms are very similar, making it difficult to detect the local vibrational mode of $\mathrm{P}$ atoms in SiNWs by Raman scattering measurements. Electron spin resonance (ESR) is a sensitive method for investigating $\mathrm{P}$ donors or conduction electrons in $\mathrm{Si}^{9,10}$ It is also sensitive to defects with dangling bonds. This method makes it possible to obtain information on both $\mathrm{P}$ donors and defects in SiNWs.

The passivation of $\mathrm{P}$ by hydrogen $(\mathrm{H})$ has been extensively studied for P-doped bulk Si. ${ }^{11-15}$ When $\mathrm{H}$ atoms are introduced into $\mathrm{P}$-doped $\mathrm{Si}$, the $\mathrm{H}$ atoms locate at the antibonding site of the nearest $\mathrm{Si}$ neighbor to a $\mathrm{P}$ atom, forming a $\mathrm{P}-\mathrm{Si}-\mathrm{H}$ passivation center. ${ }^{11-15}$ This effect can be used to control the active carrier concentration in SiNWs. Indeed, we have proved the $\mathrm{H}$ passivation of $\mathrm{B}$ in SiNWs in our previous paper. ${ }^{8}$ Defects with dangling bonds can be also passivated by hydrogen. Interfacial defects between the surface oxide and $\mathrm{Si}$ core of SiNWs inevitably exist and affect the electrical properties of SiNWs. The control of interfacial defects by powerful techniques such as $\mathrm{H}$ passivation is also an impor-

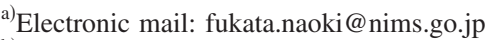

b) Also at Special Research Project on Nanoscience, University of Tsukuba, 1-1-1 Tennodai, Tsukuba 305-8573, Japan.
}

tant subject for the realization of nanoscale silicon devices using SiNWs.

In this study, $\mathrm{P}$ doping was performed during the formation of SiNWs by laser ablation of a Si target with $\mathrm{P}$ and nickel (Ni) atoms. The former acts as a dopant impurity and the latter as a catalyst. The states of $\mathrm{P}$ atoms in SiNWs were investigated by ESR at low temperatures and energydispersive $\mathrm{x}$-ray spectroscopy (EDX) measurements. These results showed that $\mathrm{P}$ atoms were doped in the substitutional sites in crystalline $\mathrm{Si}$ core of SiNWs and were activated in the sites. We also investigated the passivation effect of $\mathrm{P}$ donors and defects in P-doped SiNWs by $\mathrm{H}$ and oxygen treatments.

P-doped SiNWs were synthesized at $1200{ }^{\circ} \mathrm{C}$ in flowing argon (Ar) gas at 50 SCCM (SCCM denoter cubic centimeter per minute at STP) by catalytic laser ablation of a $\mathrm{Si}$ target with $\mathrm{Ni}_{2} \mathrm{P}$ compounds, namely, $\mathrm{Si}_{100-x}\left(\mathrm{Ni}_{2} \mathrm{P}\right)_{x}(x$ $=0.5,5$, and 10) targets. For comparison, a $\mathrm{Si}_{99} \mathrm{Ni}_{1}$ target was used for the synthesis of undoped SiNWs. The growth is conducted by the so-called vapor-liquid-solid mechanism. ${ }^{16}$ A frequency-doubled $\mathrm{Nd}$ doped yttrium aluminum garnet laser (532 nm, $7 \mathrm{~ns}$ pulse width, $10 \mathrm{~Hz}, 150 \mathrm{~mJ} /$ pulse) was used to ablate the targets. SiNWs were directly deposited on a Si substrate, then transferred to a quartz capsule for ESR measurements or a molybdenum (Mo) grid for electron microscopy with EDX measurements. ESR measurements were carried out at $4.2 \mathrm{~K}$ using an $X$-band ESR spectrometer with a magnetic field modulation of $100 \mathrm{kHz}$ to investigate the state of the $\mathrm{P}$ donor in SiNWs. Scanning transmission electron microscopy (STEM) (Hitachi: S-5500, $30 \mathrm{kV}$; JEOL: JEM3000F, $300 \mathrm{kV}$ ) and transmission electron microscopy (TEM) (JEOL: JEM4000EX, $400 \mathrm{kV}$ ) were used to observe SiNWs and to investigate the details of their structures. EDX measurements were also performed in the TEM to confirm P doping in SiNWs. To investigate the passivation of defects in SiNWs, some specimens were thermally oxidized at $900{ }^{\circ} \mathrm{C}$ for $30 \mathrm{~min}$ in $\mathrm{O}_{2}$ gas at a pressure of 200 torr after the synthesis of SiNWs. Hydrogen atom treatments (HATs) using remote downstream high flux $\mathrm{H}$ atoms were also performed at 120 and $400{ }^{\circ} \mathrm{C}$ for $30 \mathrm{~min}$ to investigate the passivation 

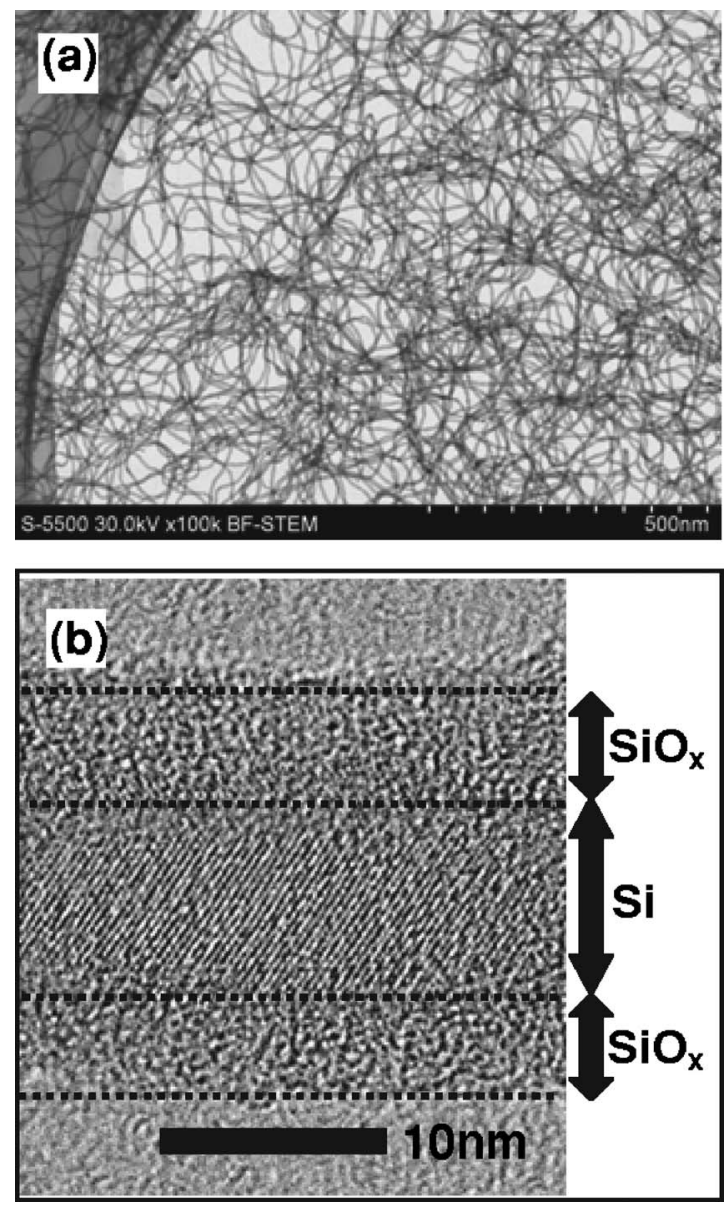

FIG. 1. Representative (a) STEM and (b) a high-resolution TEM images of the SiNWs synthesized using a $\mathrm{Si}_{99.5}\left(\mathrm{Ni}_{2} \mathrm{P}\right)_{0.5}$ target.

of both defects and P dopants. In this method, the specimens are placed in a quartz tube at a distance of $15 \mathrm{~cm}$ from the plasma source and hydrogenated only by $\mathrm{H}$ atoms. Thus, in this remote method, no damage is caused by $\mathrm{H}$ plasma. The details of the laser ablation and hydrogenation procedures are reported in Refs. 17 and 18 and in Ref. 15, respectively.

A large number of SiNWs were synthesized for ESR measurements. The STEM image of typical SiNWs synthesized using a $\mathrm{Si}_{99.5}\left(\mathrm{Ni}_{2} \mathrm{P}\right)_{0.5}$ target is shown in Fig. 1(a). The high-resolution TEM image is shown in Fig. 1(b). The Si lattice fringes are seen inside the SiNW, showing that the SiNW is sheathed in an amorphous $\mathrm{SiO}_{x}(x \leqslant 2)$ layer and the core is crystalline $\mathrm{Si}$. The average $\mathrm{Si}$ core diameter of SiNWs synthesized using a $\mathrm{Si}_{99.5}\left(\mathrm{Ni}_{2} \mathrm{P}\right)_{0.5}$ target was about 5-7 nm.

EDX analyses were performed for individual P-doped SiNWs. A typical result is shown in Fig. 2. The P $K \alpha$ peak was observed at $2.01 \mathrm{keV}$. The peak at $1.74 \mathrm{keV}$ is due to the Si $K \alpha$ line, and the peak at $2.29 \mathrm{keV}$ is due to the Mo $K \alpha$ line caused by the Mo grid. This result indicates the presence of $\mathrm{P}$ atoms in the SiNW, including the surface oxide layer. The content of P in SiNWs is roughly estimated to be in the order of $1-7$ at. \% from the peak intensity; this is in agreement with the values deduced from the ESR measurements.

ESR measurements were performed to further investigate the P-doping sites in SiNWs. Figure 3(a) shows the typical results of ESR measurements for undoped and P-doped SiNWs synthesized using $\mathrm{Si}_{99} \mathrm{Ni}_{1}$ and $\mathrm{Si}_{95}\left(\mathrm{Ni}_{2} \mathrm{P}\right)_{5}$ targets, respectively. The ESR signal observed for P-doped SiNWs is deconvoluted at least to three components with $g$ Downloaded 24 Dec 2009 to 130.158.56.127. Redistribution subject

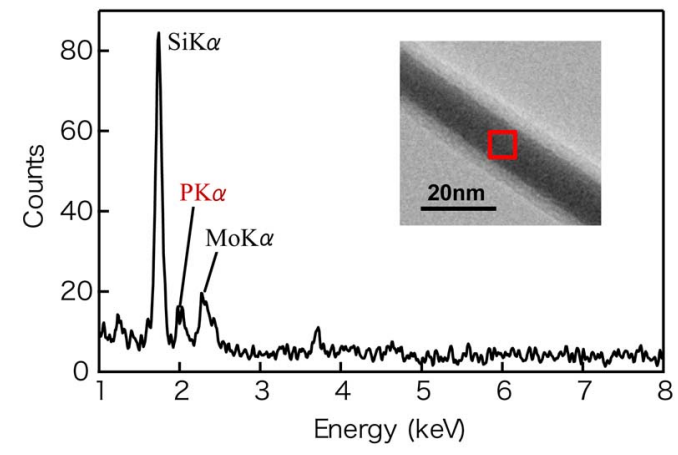

FIG. 2. (Color online) EDX spectra observed for SiNWs synthesized using a $\mathrm{Si}_{90}\left(\mathrm{Ni}_{2} \mathrm{P}\right)_{10}$ target. The STEM image in the inset shows the analysis point in a P-doped SiNW.

values of 1.998, 2.002, and 2.005, while no component with a $g$ value of 1.998 was observed for undoped SiNWs. The $g$ value of 1.998 corresponds to that of conduction electrons in $\mathrm{Si}$, indicating that the ESR signal can be attributed to the conduction electrons in the Si core of the SiNWs, and that $\mathrm{P}$ atoms were clearly doped in substitutional sites of the crystalline Si core during laser ablation. Figure 3(b) shows the dependence of the peak-to-peak width of the ESR signal of the conduction electrons on the $\mathrm{Ni}_{2} \mathrm{P}$ content in the target. The ESR signal width of conduction electrons depends significantly on the $\mathrm{P}$ concentration, i.e., it increases with increasing $\mathrm{Ni}_{2} \mathrm{P}$ content in the target. A similar dependence was observed in heavily P-doped bulk Si. It can be explained by an increase in scattering among conduction electrons and with the $\mathrm{P}$ atoms, resulting in a decrease in the spin-lattice relaxation time $T_{1}$, leading to a broadening of the signal width with increasing P concentration. ${ }^{9,10}$ Assuming that the data in P-doped SiNWs correspond to those in bulk $\mathrm{Si}$, the concentration of $\mathrm{P}$ in SiNWs is estimated to probably reach about $10^{20} \mathrm{~cm}^{-3}$.

The other ESR signals at 2.002 and 2.005 are related to certain defects. Similar signals were observed in SiNWs. ${ }^{19,20}$ These results suggest that the former is due to defects in the surface oxide of SiNWs called $E X$ centers, ${ }^{19,20}$ and the latter is probably attributable to interfacial defects between the sur-

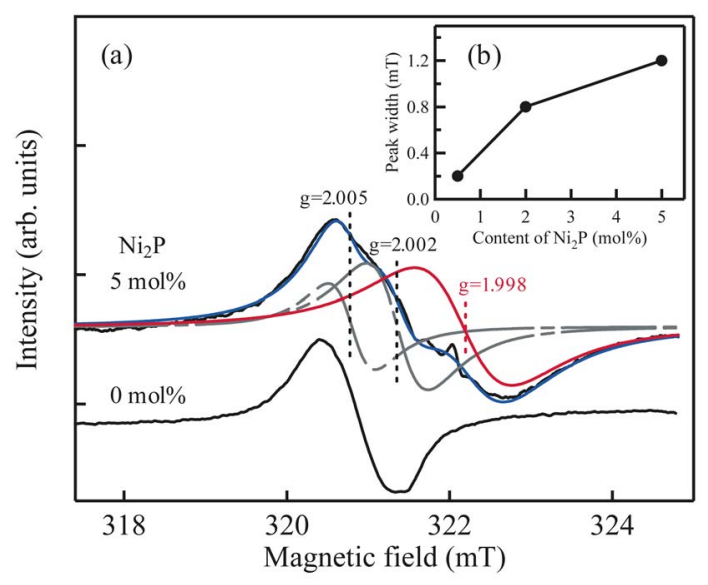

FIG. 3. (Color online) (a) ESR spectra observed for undoped and P-doped SiNWs synthesized using $\mathrm{Si}_{99} \mathrm{Ni}_{1}$ and $\mathrm{Si}_{95}\left(\mathrm{Ni}_{2} \mathrm{P}\right)_{5}$ targets, respectively. The ESR signal for P-doped SiNWs was deconvoluted into three components. (b) Dependence of the peak-to-peak width of the ESR signal of the conduction electrons on the $\mathrm{Ni}_{2} \mathrm{P}$ content in the target. 

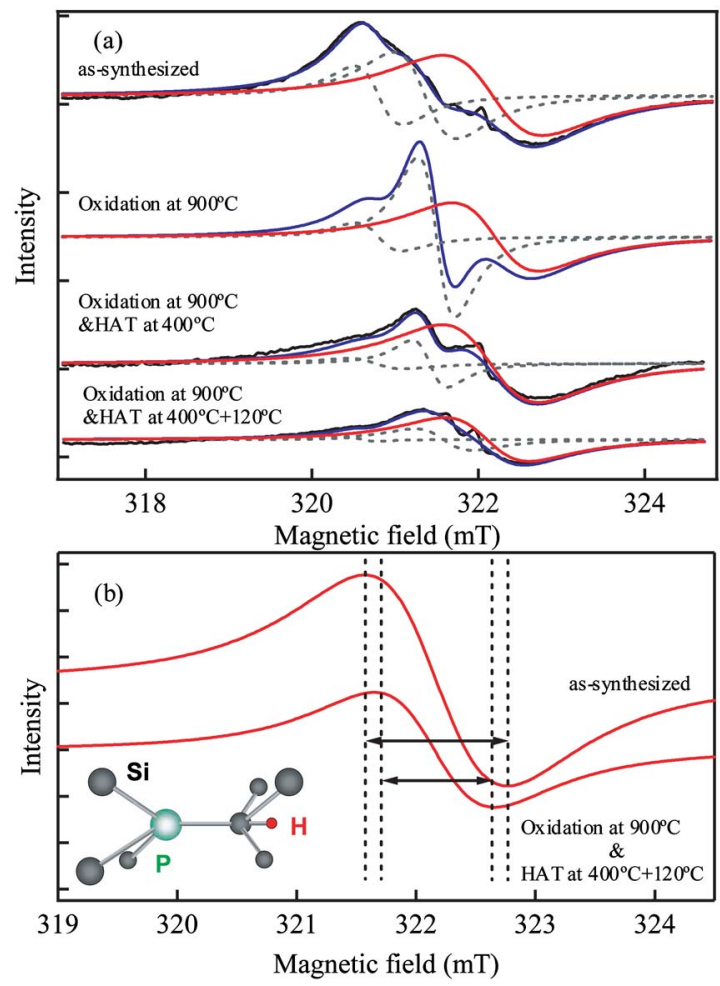

FIG. 4. (Color online) (a) Dependence of ESR signals on oxidation and HAT temperature, and (b) change in the ESR signal of conduction electrons by oxidation and HAT. The SiNWs were synthesized using a $\mathrm{Si}_{95}\left(\mathrm{Ni}_{2} \mathrm{P}\right)_{5}$ target.

face oxide and $\mathrm{Si}$ core of SiNWs which are so-called $P_{b}$ centers. We also observed a similar signal for nanocrystalline $\mathrm{Si}$ embedded in $\mathrm{SiO}_{2}{ }^{21}$ Wang reported that amorphous $\mathrm{Si}$ states exist around the interface between the surface oxide and $\mathrm{Si}$ core of SiNWs, and defects in such amorphous $\mathrm{Si}$ states can be the origin of the signal at $2.005 .{ }^{20}$ It is, however, not clear at the present time.

Next, the passivation of defects and P donors in SiNWs was investigated, as shown in Fig. 4. Annealing in $\mathrm{O}_{2}$ gas at $900{ }^{\circ} \mathrm{C}$ reduced the ESR signal intensity of interfacial defects $(g=2.005)$, indicating that oxidation is effective against their passivation. On the other hand, the ESR signal at 2.002 increases rather than decreases. For further passivation of the interfacial defects and $E X$ centers, $\mathrm{H}$ atoms were introduced into P-doped SiNWs by HAT at $400{ }^{\circ} \mathrm{C}$. The interfacial defects were almost completely passivated by hydrogenation. The intensity of the $E X$ centers decreased by more than twothirds, showing that hydrogenation is effective against the passivation of the $E X$ centers.

The ESR signal of conduction electrons showed no change after oxidation at $900{ }^{\circ} \mathrm{C}$ and subsequent $\mathrm{HAT}$ at $400{ }^{\circ} \mathrm{C}$. In general, $\mathrm{H}$ passivation of $\mathrm{P}$ donors, i.e., the decrease in the concentration of conduction electrons, is achieved by hydrogenation at less than $200{ }^{\circ} \mathrm{C}$. The most appropriate temperature is around $120^{\circ} \mathrm{C}$, which is due to the thermal stability of the $\mathrm{P}-\mathrm{Si}-\mathrm{H}$ passivation center. ${ }^{12,15}$ To investigate the $\mathrm{H}$ passivation of $\mathrm{P}$ donors, HAT was performed at $120{ }^{\circ} \mathrm{C}$ after HAT at $400{ }^{\circ} \mathrm{C}$. The results are also shown in Fig. 4(a). The interfacial defects and $E X$ centers were further passivated by HAT at $120{ }^{\circ} \mathrm{C}$. The change in the ESR signal of conduction electrons is shown in Fig. 4(b). It has been reported that the ESR signal intensity of conduction electrons shows no linearity with $\mathrm{P}$ concentration in heavily P-doped specimens, ${ }^{10}$ i.e., the reduction of active carrier concentration by HAT cannot be estimated from the change in intensity. Instead of using intensity, the passivation efficiency can be estimated from the ESR linewidth, which closely depends on P concentration. ${ }^{9,10}$ If the ESR linewidth decreased for heavily P-doped $\mathrm{Si}$, it would indicate the passivation of $\mathrm{P}$ by H. Figure 4(b) clearly shows this, giving evidence for the passivation of $\mathrm{P}$ by $\mathrm{H}$. If the data reported for bulk Si are assumed to correspond to those for SiNWs, it can be estimated that $30 \%$ of active P dopants were passivated by HAT at $120^{\circ} \mathrm{C} .{ }^{9,10}$

In conclusion, $\mathrm{P}$ doping in SiNWs during laser ablation was confirmed by the ESR signal of conduction electrons and the EDX peak of the $\mathrm{P} K \alpha$ line. The concentration of $\mathrm{P}$ donors was controlled by the content of $\mathrm{P}$ in the $\mathrm{Si}$ target. The donor concentration of electrically active donors was also controlled by hydrogen passivation. In addition to the ESR signal of conduction electrons, the ESR results showed the presence of defects in the surface oxide layer and at the interface between the surface oxide and crystalline Si core. The defects were also passivated by hydrogen and oxygen atoms.

This work was partly supported by a Grant-in-Aid for Scientific Research (2005) on Young Scientists (B) (17760003) and the 21st Century COE (Center of Excellence) Program "Promotion of Creative Interdisciplinary Materials Science for Novel Functions" under the Ministry of Education, Culture, Sports, Science and Technology (MEXT) of Japan. TEM observations were also partly supported by MEXT's "Nanotechnology Support Project." This work was also performed under the interuniversity cooperative Research Program of the Institute for Materials Research, Tohoku University.

${ }^{1}$ Y. Cui, X. Duan, J. Hu, and C. M. Lieber, C. R. Math. 104, 5213 (2000).

${ }^{2}$ Y. Cui and C. M. Lieber, Science 291, 851 (2001).

${ }^{3}$ D. D. D. Ma, C. S. Lee, and S. T. Lee, Appl. Phys. Lett. 79, 2468 (2001).

${ }^{4}$ K. K. Lew, L. Pan, T. E. Bogart, S. M. Dilts, E. C. Dickey, J. M. Redwing, Y. Wang, M. Cabassi, T. S. Mayer, and S. W. Novak, Appl. Phys. Lett. 85, 3101 (2004).

${ }^{5}$ L. Pan, K. K. Lew, J. M. Redwing, and E. C. Dickey, J. Cryst. Growth 277, 428 (2005)

${ }^{6}$ Y. Yang, K. K. Lew, T. T. Ho, L. Pan, S. W. Novak, E. C. Dickey, J. M. Redwing, and T. S. Mayer, Nano Lett. 5, 2139 (2005).

${ }^{7}$ C. Yang, Z. Zhong, and C. M. Lieber, Science 310, 1304 (2005).

${ }^{8}$ N. Fukata, J. Chen, T. Sekiguchi, N. Okada, K. Murakami, T. Tsurui, and S. Ito, Appl. Phys. Lett. 89, 203109 (2006).

${ }^{9}$ S. Maekawa and N. Kinoshita, J. Phys. Soc. Jpn. 20, 1447 (1965).

${ }^{10}$ J. D. Quirt and J. R. Marko, Phys. Rev. B 5, 1716 (1972).

${ }^{11}$ K. J. Chang and D. J. Chadi, Phys. Rev. Lett. 60, 1422 (1988).

${ }^{12}$ K. Bergman, M. Stavola, S. J. Pearton, and T. Hayes, Phys. Rev. B 38, 9643 (1988).

${ }^{13}$ S. K. Estreicher, L. Throckmorton, and D. S. Marynick, Phys. Rev. B 39, 13241 (1989).

${ }^{14}$ C. Herring, N. M. Johnson, and C. G. van de Walle, Phys. Rev. B 64, 125209 (2001)

${ }^{15}$ N. Fukata, S. Sasaki, S. Fujimura, H. Haneda, and K. Murakami, Jpn. J. Appl. Phys., Part 1 35, 3937 (1996).

${ }^{16}$ R. S. Wagner and W. C. Ellis, Appl. Phys. Lett. 4, 89 (1964).

${ }^{17}$ N. Fukata, T. Oshima, K. Murakami, T. Kizuka, T. Tsurui, and S. Ito, Appl. Phys. Lett. 86, 213112 (2005).

${ }^{18}$ N. Fukata, T. Oshima, N. Okada, T. Kizuka, T. Tsurui, S. Ito, and K. Murakami, J. Appl. Phys. 100, 024311 (2006).

${ }^{19}$ A. Baumer, M. Stutzmann, M. S. Brandt, F. C. K. Au, and S. T. Lee, Appl. Phys. Lett. 85, 943 (2004).

${ }^{20}$ R. P. Wang, Appl. Phys. Lett. 88, 142104 (2006).

${ }^{21}$ N. Fukata, C. Li, H. Morihiro, K. Murakami, M. Mitome, and Y. Bando, Appl. Phys. A: Mater. Sci. Process. 84, 395 (2006).

o AIP license or copyright; see http://apl.aip.org/apl/copyright.jsp 